$>$ médecine/sciences $(\mathrm{m} / \mathrm{s})$ a, tout au long de son existence, accompagné les nombreux débats qui ont agité la communauté scientifique et médicale concernant les questions de bioéthique posées par les bouleversements majeurs que les avancées de la recherche biomédicale ont introduits dans les pratiques thérapeutiques. La révision des lois de bioéthique est désormais proche et notre revue a voulu éclairer nos lectrices et nos lecteurs en leur proposant des regards multiples et croisés sur différents thèmes que cette révision aborde. Regard d'une juriste sur l'histoire de ces lois, leur genèse et les débats qui ont présidé à leur évolution jusqu'à ce jour, regard d'une sociologue sur certains des débats qui ont animé les États généraux de 2018, regard d'un philosophe penseur du «moment » et s'interrogeant sur ce que signifie la bioéthique, et, enfin, regards comparatifs de collègues étrangers mais francophones (un grand merci à ces auteur[e]s !) qui nous éclairent sur la façon dont certaines des thématiques faisant l'objet de ces lois dans notre pays sont abordées dans d'autres pays. Enfin, nous essaierons, autant que faire se peut, d'accompagner ces manuscrits de Nouvelles et de Synthèses portant sur les dernières avancées des sciences biologiques et médicales susceptibles d'éclairer les débats en leur apportant des données scientifiques et cliniques.

Marie Gaille et la Rédaction <

La dernière loi relative à la bioéthique date du 7 juillet 2011 . Elle a prévu que «Tout projet de réforme sur les problèmes éthiques et les questions de société soulevés par les progrès de la connaissance dans les domaines de la biologie, de la médecine et de la santé doit être précédé d'un débat public sous forme d'états généraux » organisé à l'initiative du Comité consultatif national d'éthique pour les sciences de la vie et de la santé (CCNE). Dès l'origine, avec sa création en 1983, deux idées centrales vont servir de fil conducteur à l'adoption des lois de bioéthique: (1) la nécessité d'une adaptation régulière des règles juridiques aux nouvelles applications issues de la recherche en s'interrogeant aussi bien sur leur acceptabilité morale et sociale que sur leurs risques, et (2) celle d'assurer l'expression la plus ouverte des opinions pluralistes en vue de dégager les consensus les plus larges possibles. Cela explique que, de 1994, date des premières lois de bioéthique, jusqu'à aujourd'hui, l'adoption de ces textes se soit appuyée

Vignette (Photo @ Inserm - Patrick Delapierre).

\section{Les lois françaises de bioéthique}

\section{Leur construction,}

leurs évolutions

\section{Dominique Thouvenin}

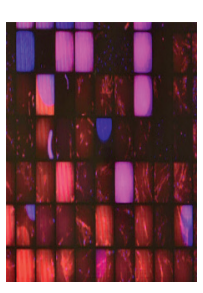

Professeure émérite de droit privé et sciences criminelles, Institut des Sciences Juridique et Philosophique de la Sorbonne (ISJPS), UMR 8103, 9 , rue Malher, 75181 Paris Cedex 4, France.

dominique.thouvenin@ehesp.fr

sur la consultation préalable d'institutions, dont le nombre n'a cessé de croître au fil des années. Et depuis la précédente révision de 2011 , il est dorénavant fait appel à la participation des citoyens, pour que le débat ne soit pas réservé aux seuls experts.

Pour comprendre, aussi bien les types de questions traitées par ces lois que leur fonction, nous aborderons successivement les points suivants: pourquoi a-t-il été indispensable d'adopter des lois? Quelle a été leur construction initiale? Comment est-on passé de trois lois (votées en 1994) à la loi du 6 août 2004 «relative à la bioéthique »? Quels ont été les enjeux du réexamen de cette loi ? Quel est le contexte actuel de l'adoption de la future loi ?

\section{Pourquoi a-t-il été indispensable d'adopter des lois?}

Ce sont les applications technologiques de découvertes effectuées grâce à la recherche biomédicale qui ont nécessité l'adoption des lois dites « bioéthiques » du fait des transformations qu'elles ont fait subir à la fonction thérapeutique traditionnelle. Les pouvoirs publics ont choisi d'institutionnaliser le débat sur ces questions en confiant au CCNE en $1983^{1}$ une réflexion éthique en amont des choix politiques. Le lien existant entre la bioéthique et la recherche a été mis en exergue en le chargeant de donner des avis «sur les problèmes moraux qui sont soulevés par la recherche dans les domaines de la biologie, de la médecine et de la santé ».

\footnotetext{
${ }^{1}$ Décret n 83-132 du 23 février 1983, J0, 25 fév. 1983.
} 
Une fois le terrain défriché par le CCNE qui avait fait émerger les différentes questions qui se posaient, il fut décidé de passer «de l'éthique au droit » pour adapter notre droit, voire créer des normes juridiques inédites ${ }^{2}$. Mais cette entreprise s'avéra particulièrement difficile: pas moins de huit années s'écoulèrent depuis la remise du premier rapport, première ébauche des textes à promouvoir, et pas moins de cinq rapports et un peu plus de deux années de discussion et de navettes parlementaires, jusqu'à l'adoption en 1994 des lois dites de bioéthique. Ce retard est en grande partie dû à la fois à de très vifs désaccords sur les questions ayant trait à l'assistance médicale à la procréation $\left(A M P^{3}\right)$ et à l'illusion que les règles juridiques ne soient que l'habillage des règles éthiques.

Tel n'est pas le cas, car il ne s'agit pas de dire comment se conduire face à telle ou telle situation, mais de fixer les conditions des relations entre des personnes dans la mesure où sont en jeu des pratiques qui, à défaut d'être organisées par la loi, n'auraient pas de légitimité. Celles-ci impliquent soit une atteinte à l'intégrité physique, soit une atteinte à la vie privée de la personne requérant le vote de lois conformément à la Constitution du 4 octobre $1958^{4}$. II en est ainsi des activités médicales (prélèvement d'éléments du corps humain sur une personne portant atteinte à l'inviolabilité de son corps pour apporter des soins à des malades qui en ont besoin, tout comme le recueil, la transmission et le traitement d'informations nominatives dans l'intérêt de la recherche en santé entraînant une violation du secret professionnel médical) qui, lorsqu'elles ne sont pas réalisées dans l'intérêt thérapeutique de la personne, constituent des infractions pénales, sauf si une loi prévoit à quelles conditions celles-ci peuvent ne pas être punies.

La possibilité de tirer des informations identifiantes à partir d'un prélèvement biologique, parce que susceptible de porter atteinte à la vie privée, nécessitait également l'adoption d'une loi, sachant que toutes les pratiques en question ne sont pas nécessairement médicales (ce qui est le cas du recours aux empreintes génétiques dans les contentieux de filiation ainsi que dans le procès pénal).

Enfin, la protection des informations, aussi bien nominatives que génétiques, issues des pratiques de soins et de recherche, devait être assurée en conformité aux principes de la protection de la vie privée et de la loi «Informatique et Libertés».

Telles sont les règles constitutionnelles françaises régissant l'élaboration des lois. Encore faut-il préciser les modalités de construction du projet global retenues par le pouvoir politique qui constituent un outil précieux de compréhension de la manière dont certaines questions continuent d'être discutées aujourd'hui.

${ }^{2}$ Conseil d'État, Sciences de la vie, De l'éthique au droit, La Documentation française, Notes et Études documentaires, 1988, p. 7.

${ }^{3}$ C'est l'expression « assistance médicale à la procréation (AMP) 》 qui a été retenue tout au long de cet article; en effet, c'est cette dernière qui, en définitive, a été adoptée par la loi alors que le projet de loi $n^{\circ} 2601$ avait choisi celle de «procréation médicalement assistée ». Le passage d'une formulation à l'autre est dû au souhait des parlementaires que l'accent ne soit pas mis sur le résultat obtenu (la procréation) mais sur l'intervention médicale (l'assistance médicale) nécessaire à l'obtention de ce résultat espéré.

${ }^{4}$ Art. 34 de la Constitution du 4 octobre 1958. Cette constitution est le texte fondateur de la Ve République (http://www.vie-publique.fr/IMG/pdf/La_constitution-1958-web.pdf).

\section{Quelle est la construction initiale des lois dites de bioéthique de 1994 ?}

Trois projets de loi furent déposés devant l'Assemblée Nationale le 22 mars 1992 selon l'architecture suivante:

- tout d'abord, des dispositions introduites dans le Code civil énonçant «les principes généraux qui fondent le statut juridique du corps humain pour assurer le respect de la dignité de la personne et protègent l'intégrité du patrimoine génétique, et à travers lui, l'espèce humaine » et fixant « un cadre juridique à l'utilisation des tests génétiques et des tests d'identification génétique, afin de préserver les droits fondamentaux de la personne $\gg^{5}$;

- puis des règles intégrées dans le Code de la santé publique relatives au don et à l'utilisation des éléments et produits du corps humain et à la procréation médicalement assistée ${ }^{6}$ qui répondent à «une évolution notable de la médecine » consistant à mettre « en œuvre des techniques de substitution pour pallier les dysfonctionnements de mécanismes vitaux $\gg^{7}$;

- enfin, des dispositions régissant le traitement de données nominatives à des fins de recherche en santé 8 et transcrites dans la loi $n^{\circ} 78-17$ du 6 janvier 1978 relative à l'informatique, aux fichiers et aux libertés, dans l'objectif de «concilier le droit des personnes à la protection de l'intimité de leur vie privée avec le développement nécessaire des connaissances médicales $\gg^{9}$.

Les règles issues de ces trois lois adoptées en 1994 relèvent de deux grandes catégories de dispositions:

- d'une part, des règles générales, souvent présentées comme constituant les «grands principes», qui reconnaissent des droits généraux à toute personne et qui visent à la protéger : «la primauté de la personne humaine», le «respect de l'être humain dès le commencement de sa vie », l'inviolabilité et l'indisponibilité du corps humain, ainsi que "l'intégrité de l'espèce humaine » et l'absence de «discrimination en raison de ses caractéristiques génétiques »;

- d'autre part, des règles spécifiques qui organisent des pratiques médicales dans l'intérêt d'autrui ou

\footnotetext{
${ }^{5}$ Projet de loi, Assemblée nationale, $n^{\circ} 2599,25$ mars 1992.

${ }^{6}$ Projet de loi, Assemblée nationale, n² 2600, 25 mars 1992.

7 L'exposé des motifs du projet de loi $n^{\circ} 2600$ déposé à l'Assemblée nationale donne comme exemples les «greffes d'organes, qui impliquent de remplacer un organe malade par un organe sain prélevé sur le corps d'un tiers, vivant ou mort», «la thérapie génique qui s'apparente, en l'état actuel des connaissances, à une sorte de greffe moléculaire », les techniques de «procréation médicalement assistée » qui constitue «le domaine privilégié de cette médecine de substitution».

${ }^{8}$ Projet de loi, Assemblée nationale, $n^{\circ} 2601,25$ mars 1992.

${ }^{9}$ Conseil des ministres du 18 décembre 1991 sur les orientations du Gouvernement
} en matière d'éthique biomédicale. 
dans celui de la recherche et qui, pour ce faire, nécessitent de porter atteinte soit à l'intégrité physique, soit à la vie privée, ventilées en fonction de ces deux champs.

Cet ensemble de règles présente trois caractéristiques essentielles. La première réside dans la prise en compte des deux catégories d'intérêts que ces deux corps de règles ont pour fonction avérée de ménager: ceux de toute personne, grâce aux droits subjectifs qui lui sont reconnus dans le Code civil au titre de ce que l'on appelle le «statut du corps humain »; et ceux des patients dont les soins nécessitent des éléments biologiques du corps humain, de même que ceux des personnes dont l'accès aux informations de nature génétique est autorisé pour des motifs de preuve ou de diagnostic. Les règles du Code de la santé publique fixent à la fois les conditions d'exercice de ces activités par les praticiens et celles de leur accès pour les patients qui en ont besoin ; les règles intégrées dans la loi Informatique et Liberté autorisent les chercheurs, dans l'intérêt du développement des connaissances, à accéder aux données de santé nominatives auprès des médecins traitants, malgré le secret médical auquel ces derniers sont astreints. Aussi les «grands principes» sont-ils moins une protection en soi que le moyen d'établir les exceptions autorisant l'accès, pour les personnes qui en ont besoin, au corps biologique d'une autre personne ainsi que pour les chercheurs aux données médicales de personnes qu'ils ne soignent pas.

La seconde de ces caractéristiques tient aux «modifications subies par le projet de loi modifiant le code de la santé publique (CSP) », organisant toutes les formes de médecine de substitution. II réunissait dans un livre unique les règles relatives aux éléments et produits du corps humain ainsi qu'à la création et la conservation d'embryons humains en vue de la procréation; mais un amendement ajouta la possibilité pour ces derniers, qu' «à titre exceptionnel, [...] un projet de recherche soit effectué ». C'est clairement cette possibilité de changement qui a conduit le Sénat à critiquer "l'architecture initiale du projet de loi, fondée sur l'inclusion dans un même livre du code de la santé publique des dispositions consacrées au don et à l'utilisation des éléments et produits du corps humain, à la procréation médicalement assistée », parce qu'elle «consacre un principe d'instrumentalisation du corps humain ».

Le Sénat scinda les dispositions légales en les répartissant de la manière suivante: les règles régissant I'AMP furent insérées dans le livre II du Code de la santé publique consacré à "l'action sanitaire et médico-sociale en faveur de la famille, de l'enfance et de la jeunesse », la référence à l'AMP étant supprimée de l'intitulé du livre VI, ce dernier ne portant plus que sur les éléments et produits du corps humain. Il entendit ainsi écarter explicitement l'assimilation des embryons humains à des produits du corps humain, laquelle méconnaîtrait leur vocation à devenir des êtres humains. Aussi l'intégration, dans le livre II du Code de la santé publique, des textes les concernant a-t-il traduit le souci du Sénat de mettre en avant l'objectif à atteindre : la conception d'un enfant, d'où le lien avec la famille et l'enfance. En outre, il vota un texte interdisant la recherche sur l'embryon en prévoyant toutefois que des «études » puissent être autorisées «à condition qu'elles n'entraînent ni la destruction de l'embryon, ni des amputations ou des lésions irréversibles ».

La troisième de ces caractéristiques concerne «le traitement des données nominatives de santé » qui, bien que posant des questions sensibles de protection de la vie privée, sera rapidement perdu de vue et négligé.

C'est sur l'accès au corps humain biologique et encore plus sur l'accès à l'AMP et l'utilisation de l'embryon humain (uniquement pour permettre à un couple d'avoir des enfants ou bien, également à des fins de recherche, lorsque le couple ne souhaite plus en avoir) que se concentreront débats, contestations et revendications de modifications des règles existantes.

\section{Comment est-on passé de trois lois à la loi du 6 août 2004 « relative à la bioéthique »?}

Seul le réexamen de la loi modifiant le Code de la santé publique ayant été prévu, son champ a été restreint à celui des domaines relatifs aux pratiques médicales de médecine prédictive, d'identification des maladies d'origine génétique, de recherche génétique, de prélèvement et d'utilisation des éléments et produits du corps humain, de diagnostic prénatal, d'AMP, et de recherche sur l'embryon et les cellules embryonnaires. La loi aurait dû être réexaminée dans un délai maximum de cinq ans après son entrée en vigueur, soit au plus tard en 1999 et faire l'objet d'une évaluation par l'Office parlementaire d'évaluation des choix scientifiques et technologiques (OPECST). Or celui-ci l'effectua dans le contexte du clonage en 1996 de la brebis Dolly ${ }^{10}$, puis dans celui de l'identification en 1998 des premières lignées de cellules souches embryonnaires humaines. Cette dernière souleva la question de savoir si des embryons créés en vue d'une procréation, lorsqu'ils ne seront plus utilisés dans cette finalité, peuvent l'être pour une autre finalité, celle de la recherche sur l'embryon.

Le gouvernement de l'époque, craignant d'être confronté à nouveau à des débats houleux au sujet de l'embryon, alors que la perspective d'une utilisation potentielle des cellules souches embryonnaires pour soigner des maladies avait changé la donne, ne déposa le projet de loi que le 20 juin 2001 , lequel aboutit à la loi relative à la bioéthique du 6 août 2004. L'attention s'est focalisée sur les pratiques de reproduction humaine, car celles-ci étaient et

10 II s'agit du clonage reproductif par transfert d'un noyau somatique dans l'ovocyte énucléé d'une brebis, l'embryon ainsi obtenu étant transféré dans l'utérus d'une autre brebis et se développant jusqu'à la naissance. 
sont encore l'objet de très vives discussions dues à la disparité des valeurs et des croyances de chacun.

Les modifications apportées par la loi du 6 août 2004 ont été de trois ordres:

1. Les règles applicables aux pratiques médicales et/ou de recherche dont l'objet est le corps humain et l'embryon comme ressource ou entité biologiques ont été unifiées, ce qui s'est traduit par la création de l'Agence de la biomédecine (ABM), dont les compétences portent sur tout le vivant biologique, à savoir «la greffe, la reproduction, l'embryologie et la génétique ».

2. Alors que la légitimé de ces activités médicales était liée à l'exigence qu'elles poursuivent, la seule finalité autorisée jusqu'alors par la loi $n^{\circ} 94-654$ du 29 juillet 1994, celle de 2004 a admis la possibilité d'un changement de finalité dans un certain nombre de cas, notamment celui du couple qui, lorsqu'il n'a plus de projet parental, peut donner son accord pour que «leurs embryons » créés en vue d'une procréation «fassent l'objet d'une recherche».

3. Cette loi a maintenu l'interdiction de la recherche sur l'embryon, mais admis des dérogations pour une durée de cinq ans.

\section{Quels ont été les enjeux du réexamen de la loi du 6 août 2004 ?}

C'est le régime transitoire et dérogatoire des recherches sur l'embryon qui a imprimé sa marque sur son réexamen. Venant à échéance en février 2011 , les règles devaient être adoptées dans les délais prévus. Plusieurs types d'évaluations de nature scientifique ou relatives à l'application de la loi de 2004 (celles de l'OPECST et de I'ABM), ainsi que des études (par le Conseil d'État en vue d'une étude préalable à la révision de la loi) et avis (du CCNE pour identifier les problèmes philosophiques et les interrogations éthiques) furent rendus. Et, pour la première fois, se sont tenus des États généraux de la bioéthique «en vue d'engager un débat public faisant appel à la participation des citoyens ${ }^{11}$ dans l'objectif déclaré que le débat ne soit pas confisqué par les experts.

Les quelques modifications apportées par la loi $n^{\circ} 2011-814$ du 7 juillet 2011 l'ont été à la marge: autorisation du «don croisé d'organes »; obligation pour la personne porteuse d'une anomalie génétique grave d'en informer les membres de sa famille potentiellement concernés, lorsque des mesures de prévention ou de soins peuvent leur être proposées; possibilité pour la femme de conserver ses ovocytes pour préserver sa fertilité ultérieure; entrée dans le code civil des neurosciences et de l'imagerie médicale sous la forme d'un texte limitant l'emploi des «techniques d'imagerie cérébrale » aux seules finalités soit médicale, soit de recherche scientifique, ou aux expertises judiciaires.

Une fois de plus, les deux grands enjeux de cette loi auront été l'extension éventuelle de l'AMP à des femmes seules ou vivant en couple et la question de la recherche sur l'embryon. Fallait-il ou non

11 «La méthode et le contenu de la révision de la loi de bioéthique », Conseil des ministres du 16 juillet 2008, http://www.gouvernement.fr/gouvernement/la-methode-et-le-contenu-de-la-revision-de-laloi-de-bioethique accepter les évolutions sociales au sein de la famille et admettre I'AMP comme une aide à la parentalité ou s'en tenir au modèle construit dans les années 1980 n'autorisant son accès qu'à des couples formés d'un homme et d'une femme dans les cas d'infertilité médicalement reconnue. C'est cette règle que cette loi a maintenue. Elle l'a seulement ouverte aux couples hétérosexuels pacsés, sans qu'ils aient à justifier de deux années de vie commune.

Pour ce qui concerne l'embryon, cette loi a confirmé, en le rendant permanent, le régime d'interdiction avec dérogations à la suite d'une bataille rude qui opposa deux conceptions irréductibles: la première, se référant aux valeurs, conservait l'interdiction tout en fixant des dérogations pour marquer l'illégitimité de la recherche; la seconde, mettant en balance les intérêts entre la nécessité de conduire la recherche sur les embryons et les cellules souches embryonnaires et la protection de l'embryon, défendait sa légitimité en ne l'autorisant qu'à des conditions drastiques. Finalement, c'est une loi spécifique, la loi $n^{\circ} 2013-$ 715 du 6 août 2013, qui a autorisé, sous certaines conditions, la recherche sur l'embryon et les cellules souches embryonnaires.

\section{Quel est le contexte actuel de l'adoption de la future loi?}

La loi du 7 juillet 2011 avait prévu qu'elle ferait « l'objet d'un nouvel examen d'ensemble par le Parlement dans un délai maximal de sept ans après son entrée en vigueur », précédé d'un débat public confié au CCNE. Celui-ci a ouvert le 18 janvier 2018 la longue liste des différentes évaluations (de l'ABM en janvier 2018, de I'OPESCT en octobre 2018), études (du Conseil d'État sur sa révision en juin 2018) et avis (synthèse du CCNE sur les États généraux de la bioéthique remis le 2 juillet 2018, puis Avis 129 du 18 septembre 2018). De plus, la Mission d'information sur la révision de la loi relative à la bioéthique, après avoir mené un cycle d'auditions du 19 juillet au 7 novembre 2018 sur la révision de la loi relative à la bioéthique, devait publier son rapport en décembre. Le porte-parole du Gouvernement avait déclaré, le 18 juillet 2018 à l'issue du Conseil des ministres que « Le gouvernement présentera avant la fin de l'année un projet de loi sur les questions de bioéthique, y compris la PMA, qui sera débattu par le Parlement début $2019 »$. On relèvera avec le plus grand intérêt, que revenant sur cette annonce le 27 novembre 2018, il a précisé dans sa communication orale dans les médias que «l'extension de la procréation médicalement assistée aux couples homosexuels sera présentée 
en $2019 \gg^{12}$. C'est ainsi qu'une fois de plus, la révision de la loi de bioéthique sera polarisée sur cette question.

\section{Conclusion}

Trois points particulièrement importants peuvent être mis en exergue, à la suite de cette analyse.

1. On relèvera tout d'abord une difficulté certaine à déterminer les types d'activités relevant de la bioéthique. Elle est due dès l'origine à la séparation des règles relatives aux pratiques de soins ayant trait au corps humain de la personne et à l'AMP de celles concernant I'utilisation des données de santé dans un but de recherche. Elle a empêché une vision globale des questions de bioéthique, en ne les rapportant pas à la personne concernée, mais en l'abordant du seul point de vue des activités médicales au regard de l'évolution des nouvelles technologies. Cette situation a été aggravée par le choix du législateur de ne prévoir que le seul réexamen de la loi modifiant le code de la santé publique, réduisant ainsi les modifications éventuelles à ces dernières. Elle l'a été, en outre, par les choix du CCNE d'étendre sa compétence à des questions qui n'en relèvent pas, telles que par exemple la fin de vie. C'est ainsi que les États généraux de la bioéthique et l'Avis 129 ont abordé les problèmes liés à la fin de vie qui n'ont rien à voir avec quelque évolution technologique que ce soit ${ }^{13}$. Le CCNE a été suivi sur ce point par le Conseil d'État, ce qui a permis à l'un comme à l'autre d'opposer des questions soulevées « indépendamment des évolutions scientifiques et techniques » à celles posées par ces dernières, agrégeant à la fin de vie celles de I'AMP.

2. En ce qui concerne l'AMP, le CCNE et le Conseil d'État ont distingué celle de nature palliative considérée comme de nature thérapeutique et celle dite "sociétale » ${ }^{14}$ portant sur l'accès à I'AMP des femmes seules ou des couples de femmes homosexuelles). De même, pour I'OPECST, une telle extension est de «motivation essentiellement sociétale et non principalement scientifique ». Pourtant l'AMP est bien une application technique de découvertes effectuées grâce à la recherche. Derrière cette séparation est en jeu la qualification de ce qui est ou non thérapeutique, sachant que ce n'est jamais l'utilisation de la technique qui a posé problème, mais son acceptabilité sociale.

\footnotetext{
12 À tel point que l'ensemble des médias français n'ont pas hésité à présenter le futur projet de loi relatif à la bioéthique comme étant le projet de loi «sur l'extension de la procréation médicalement assistée à toutes les femmes 》.

${ }^{13}$ Les règles relatives à la fin de vie ne relèvent pas des dispositions issues de la loi $n^{\circ} 94-654$ du 29 juillet 1994, mais ont été édictées par la loi $n^{\circ} 2005-370$ du 22 avril 2005 relative aux droits des malades et à la fin de vie, puis par la loi $n^{\circ} 2016-87$ du 2 février 2016. Elles ont été incluses, en la modifiant, dans la loi $n^{\circ} 2002-303$ du 4 mars 2002 relative aux droits des malades et à la qualité du système de santé. Ce choix est dû au fait que les pratiques en matière de fin de vie ont pour finalité l'intérêt thérapeutique personnel du patient, tandis que celles relevant de la loi relative à la bioéthique mettent en jeu l'intérêt thérapeutique d'autrui ou celui de la recherche. Le fait d'agréger les questions relatives à la fin de vie à celles se posant dans le champ des pratiques bioéthiques a pour conséquence de mettre sur le même plan des pratiques médicales conduites dans le seul intérêt de la personne malade et des pratiques médicales caractérisées par des intérêts potentiellement en conflit. Une telle présentation n'est pas neutre car elle contribue à occulter ces derniers.

${ }^{14}$ F. Héran, Pour en finir avec « sociétal ». Rev Fr Sociologie $1991 ; 32-4: 615-21$.
}

3. Enfin, alors que l'ensemble des réflexions produites à l'occasion de la révision de la loi relative à la bioéthique l'ont été entre janvier et septembre 2018, le Président de la République a successivement présenté en mars 2018 le «Plan pour l'intelligence artificielle » dans lequel la santé occupe une place prioritaire, puis en septembre 2018 la stratégie de transformation du système de santé, intitulée «Ma santé 2022 ». Celle-ci comprend notamment la création d'un espace numérique personnel de santé pour les patients, le développement d'un «bouquet de services » digitaux pour les professionnels, un pilotage national de l'esanté renforcé. Est ensuite paru, à la mi-octobre 2018 , le rapport de la mission de préfiguration du Health Data Hub, plateforme de données de santé appelée à recueillir les données de remboursement de l'assurance maladie et des hôpitaux, celles des grandes cohortes de recherche en santé publique, les données cliniques collectées par les hôpitaux, celles issues des objets connectés, etc.

Tandis que se dessinent des transformations majeures des pratiques dans le domaine de la santé du fait des technologies du numérique, les très nombreuses questions qu'elles soulèvent ne seront pas examinées par le législateur, parce que, depuis l'origine, le réexamen n'a été prévu que pour les seules dispositions intégrées dans le code de la santé publique.

Si les règles adoptées en 1994 ont eu pour objectif d'autoriser les pratiques médicales nécessitant des manipulations sur le corps humain ou sur les mécanismes fondamentaux de la vie ainsi que d'autoriser les médecins à transmettre, dans un objectif de recherche, des données médicales concernant leurs patients, le législateur n'a pas envisagé de réexamen de cette seconde catégorie de règles qui, par ailleurs, ne relèvent pas du même corpus de règles (non pas celles du Code de la santé publique, mais celles de la loi Informatique et Libertés). Cette différence de traitement s'explique vraisemblablement par le fait, qu'à l'époque, les questions les plus sensibles étaient liées à des techniques nouvelles - telles que cryoconservation, transplantation, etc. - qui avaient permis le développement d'une médecine de la prothèse et de la substitution biologique (notamment, greffes en tous genres, AMP avec tiers «donneur»). Médecine dépendante de l'obtention d'organes et d'éléments du corps humain, qui ne se trouvent que dans le corps humain, et sont prélevés sur des personnes autres que celles bénéficiant des soins ou bien passant par la création d'embryons in vitro, elle semblait poser de plus redoutables questions sociales que celles liées à l'utilisation des données 
médicales des patients à des fins de recherche, à l'époque essentiellement épidémiologique.

Mais en 2018, la collecte, le traitement et l'emploi de millions de données de santé (données massives en santé ou «Big Data» en santé) issues de sources différentes constituent désormais une question sociale déterminante. Elles vont vraisemblablement entrầner un bouleversement majeur des pratiques dans le domaine de la santé et des notions ainsi que des principes sur lesquels elles se fondent (notion de donnée de santé, médecine fondée sur les preuves [evidence-based medicine], décision thérapeutique, responsabilité, etc.) et mettre à mal les modes de régulation actuels de la santé. $\diamond$

French bioethics laws: their construction, their evolutions

TIRÉS À PART

D. Thouvenin

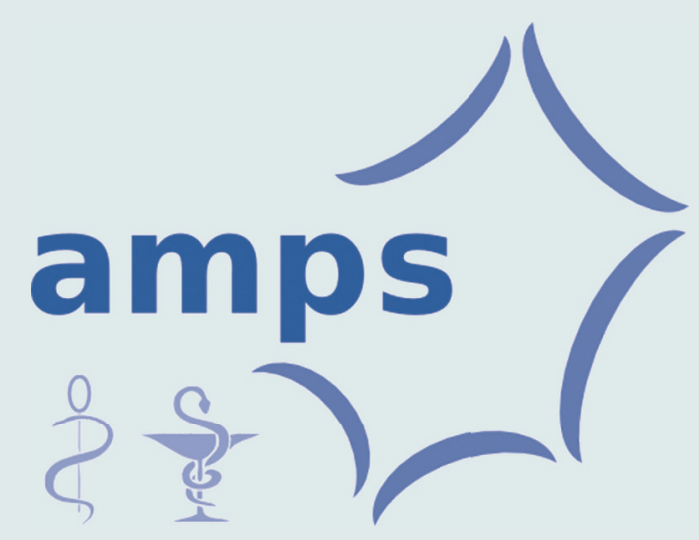

L'AMPS encourage les approches multidisciplinaires et permet aux étudiants des différentes facultés, ayant des compétences différentes, d'échanger leurs idées et d'interagir entre eux, via un groupe virtuel

(sur les réseaux sociaux) performant, des dîners doubles cursus mensuels et un congrès annuel.

Nous comptons parmi nos membres des étudiants en master, des doctorants, des internes et des cliniciens. Cette formidable diversité permet de mettre en commun les différentes expertises scientifiques et cliniques.

Elle permet également aux plus jeunes de bénéficier des conseils précieux de leurs aînés.

La newsletter, envoyée à tous les membres chaque mois, est un outil que chacun utilise au mieux.

\section{http://www.amps-asso.fr}

Groupe facebook: AMPS (Association Médecine Pharmacie Sciences)

Sur Twitter : @AssoAMPS

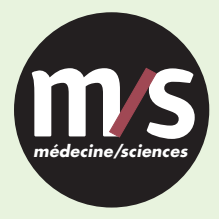

Tarifs d'abonnement $\mathrm{m} / \mathrm{s}-2019$

Abonnez-vous

à médecine/sciences
$>$ Grâce à $m / s$, vivez en direct les progrès des sciences biologiques et médicales

Bulletin d'abonnement page 86 dans ce numéro de $\mathrm{m} / \mathrm{s}$

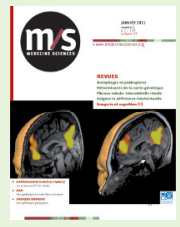

